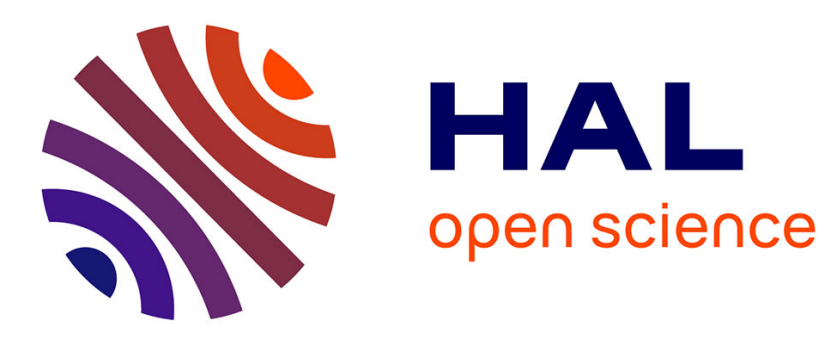

\title{
Robust Social Decisions
}

Eric Danan, Thibault Gajdos, Brian Hill, Jean-Marc Tallon

\section{To cite this version:}

Eric Danan, Thibault Gajdos, Brian Hill, Jean-Marc Tallon. Robust Social Decisions. American Economic Review, 2016, 106 (9), pp.2407 - 2425. 10.1257/aer.20150678 . halshs-01415412

\section{HAL Id: halshs-01415412 https://shs.hal.science/halshs-01415412}

Submitted on 13 Dec 2016

HAL is a multi-disciplinary open access archive for the deposit and dissemination of scientific research documents, whether they are published or not. The documents may come from teaching and research institutions in France or abroad, or from public or private research centers.
L'archive ouverte pluridisciplinaire HAL, est destinée au dépôt et à la diffusion de documents scientifiques de niveau recherche, publiés ou non, émanant des établissements d'enseignement et de recherche français ou étrangers, des laboratoires publics ou privés. 


\title{
Robust Social Decisions*
}

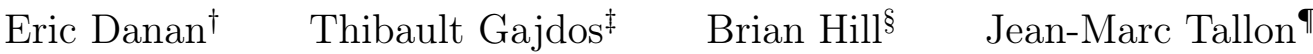

December 10, 2015

\begin{abstract}
We propose and operationalize normative principles to guide social decisions when individuals potentially have imprecise and heterogeneous beliefs, in addition to conflicting tastes or interests. To do so we adapt the standard Pareto principle to those preference comparisons that are robust to belief imprecision and characterize social preferences that respect this robust principle. We also characterize a suitable restriction of this principle. The former principle provides stronger guidance when it can be satisfied; when it cannot, the latter always provides minimal guidance.
\end{abstract}

Keywords. Unambiguous preferences, Pareto dominance, Preference aggregation, Social choice, Uncertainty

JEL Classification. D71, D81

Public policies often yield uncertain outcomes. In order to evaluate the various alternative policies and select an optimal one, policy makers need to rely on some assessment of the probabilities of these outcomes. For some critical issues such as climate change, however, this task is particularly challenging because the uncertainty at hand is not well understood enough to allow a precise assessment of the probabilities. ${ }^{1}$

${ }^{*}$ An earlier version of this paper was circulated under the title "Aggregating Tastes, Beliefs, and Attitudes under Uncertainty". We thank Marc Fleurbaey, Tzachi Gilboa, Peter Klibanoff, Fabio Maccheroni, Philippe Mongin, Sujoy Mukerji, Klaus Nehring, Efe Ok, Marcus Pivato, Xiangyu Qu, David Schmeidler, Peter Wakker, Stéphane Zuber, and three anonymous referees, as well as participants to the D-TEA 2014 meeting, DRI seminar in Paris, workshop in Bielefeld University, and seminars at Columbia University, Koç University, Queen's University Belfast, ETH-Zurich, University of Oslo, University of Warwick, Bocconi University, and HEC Montréal for useful comments and discussions. Danan thanks support from the Labex MME-DII program (ANR-11-LBX-0023-01). Gajdos thanks support from the $A^{\star}$ MIDEX project (ANR-11-IDEX-0001-02) funded by the Investissements d'Avenir program. Hill thanks support from the ANR DUSUCA (ANR-14-CE29-0003-01) and the Investissements d'Avenir program (ANR-11-IDEX-0003/Labex Ecodec/ANR-11-LABX-0047). Tallon thanks support from ANR grant AmGames (ANR-12-FRAL-0008-01) and from the Investissements d'Avenir program (ANR-10-LABX-93).

${ }^{\dagger}$ THEMA, Université de Cergy-Pontoise, CNRS, 33 boulevard du Port, 95011 Cergy-Pontoise cedex, France (eric.danan@u-cergy.fr).

${ }^{\ddagger}$ Greqam, CNRS, EHESS, Université d'Aix-Marseille (Aix Marseille School of Economics) (thibault.gajdos@univ-amu.fr).

${ }^{\S}$ GREGHEC, CNRS, HEC Paris, Université Paris-Saclay (hill@hec.fr).

TParis School of Economics, CNRS (jean-marc.tallon@univ-paris1.fr).

${ }^{1}$ Besides climate change, Henry (2006) describes two other cases - asbestos and Creutzfeld-Jacob disease in which public actions had to be (or should have been in the case of asbestos) taken on the basis of "uncertain science" (imprecise scientific knowledge). Manski (2013) discusses how relying on "incredible certitude" can mislead policy analysis and argues instead for acknowledging partial knowledge of individuals' characteristics. 
A major issue is whether there will be significant global warming - for example, of $4^{\circ} \mathrm{C}$ or more (relative to preindustrial levels) - which would have wide-ranging, and unevenly distributed, consequences on economic activity, human settlement and health around the world (IPCC, 2014). This depends on future concentrations of greenhouse gases (GHG), which themselves depend on climate policy. Both of these dependencies involve considerable uncertainty. On the one hand, climate sensitivity to GHG concentrations is imperfectly understood and cannot as yet be accurately described, even probabilistically, with full precision. Rather, a range of probabilistic models are considered plausible by climate scientists (IPCC, 2013, Section 10.8). On the other hand, the effect of a given policy on GHG concentrations depends, among other factors, on technological evolutions that are highly unpredictable and for which any prediction is essentially subjective (Stern, 2013; Pindyck, 2013). So different actors evaluating a given policy - say the French and British governments evaluating a European climate policy - may rely on different predictions and, hence, end up using different plausible ranges for the probability of global warming reaching $4^{\circ} \mathrm{C}$ under this policy - say $10 \%$ to $50 \%$ and $40 \%$ to $60 \%$, respectively. In such a situation, how should the policy be evaluated at the European level?

This paper aims at providing guidance for such policy decisions. Situations of this sort involve a "social" decision maker (the European Commission) who must choose a policy whose outcome is uncertain and affects several "individual" actors (the French and British governments). Individuals may have different utility functions - or have heterogeneous "tastes" - and consider different probabilistic models to be plausible - or have heterogeneous "beliefs". Moreover, a given individual may also consider more than one model to be plausible - or have an imprecise belief. For such an individual, which of two policies yields the highest expected utility may depend on the model considered. When a policy yields a higher expected utility than another one for all plausible models, we say that the individual unambiguously prefers the former policy to the latter. Unambiguous preferences are thus robust to belief imprecision. ${ }^{2}$

The Pareto principle is a natural guide for such decisions. We propose a robust version of this principle, requiring that if all individuals unambiguously prefer a policy to another one then so should the policy maker. We show that this unambiguous Pareto principle prescribes that the policy maker must only rely on probabilistic models that are considered plausible by all individuals. In the example above, this means that in order to guarantee that the implemented policy is unambiguously Pareto optimal, the European Commission must restrict attention to probabilities of global warming reaching $4^{\circ} \mathrm{C}$ that belong to both the French and British ranges - between $40 \%$ and $50 \%$.

As this example illustrates, the policy maker can respect unambiguous Pareto dominance even when individuals have heterogeneous beliefs, as long as these beliefs are compatible - at least one model is unanimously considered plausible. Heterogeneous yet compatible beliefs arise naturally in some contexts. ${ }^{3}$ But they are ruled out by the standard assumption that all individuals have precise beliefs - each individual considers a single probabilistic model plausible. Under

\footnotetext{
${ }^{2}$ Such preferences are also called Bewley (2002) preferences. They are incomplete expected utility preferences and are thus distinct from "robust" preferences in the sense of Hansen and Sargent (2001, 2008), which are complete non-expected utility preferences.

${ }^{3}$ For instance, if individuals' beliefs originate from partial and distinct but mutually consistent pieces of evidence, or from a common "baseline" probabilistic model that they do not fully trust.
} 
this particular assumption, we recover the well-known result that the standard Pareto principle can only be respected when all individuals have identical beliefs (Hylland and Zeckhauser, 1979; Mongin, 1995, 1998).

When individuals have incompatible beliefs - no probabilistic model is unanimously considered plausible - the unambiguous Pareto principle yields no prescription: whatever probabilistic models the policy maker takes as plausible, she may end up implementing an unambiguously Pareto dominated policy. We therefore propose restricting this principle to policies that only involve outcomes on which individual tastes are homogeneous. We show that this common-taste unambiguous Pareto principle prescribes that the policy maker must only rely on probabilistic models that are weighted averages of models considered plausible by at least some individuals. Thus this common-taste restriction provides weaker guidance than the unambiguous Pareto principle when individual beliefs are compatible - in the example above, it prescribes that the European Commission must rely on probabilities between $10 \%$ and $60 \%$. On the other hand, it still provides guidance when beliefs are incompatible - it yields the same prescription if the French range were narrowed to between $10 \%$ and $30 \%$.

Except in a few special cases, neither the unambiguous Pareto principle nor its common-taste restriction constrain the policy maker to rely on a single probabilistic model. She may do so if she wishes, but she could also rely on a range of models. ${ }^{4} \mathrm{~A}$ wider range of models results in a larger set of unambiguously optimal policies and, consequently, allows the policy maker more flexibility in selecting the policy to implement within this set. As we demonstrate, the set of unambiguously optimal policies, however large, can be computed very simply. Moreover, any policy selected within this set reflects a more or less cautious - or conservative - attitude.

Section 1 introduces the formal setup for our analysis. Section 2 contains the main results: characterizations of the unambiguous Pareto principle and its common-taste restriction. Section 3 presents additional results on computing the set of unambiguous optima and making a selection within this set. Section 4 discusses related literature. Proofs are gathered in the Appendix.

\section{Setup}

\subsection{Social decisions}

Consider a society made of a finite number $n$ of individuals. Let $S$ be a finite set of states of the world and $X$ be a set of outcomes. Society (the social decision maker) has to choose an act (a policy) $f$ whose outcome $f(s) \in X$ depends on which state $s \in S$ will occur. Let $F$ denote the set of all acts, that is all functions $f: S \rightarrow X$. We identify an outcome $x \in X$ with the constant act yielding outcome $x$ no matter which state occurs, thus viewing $X$ as a subset of $F$.

An element of $X$ specifies an outcome for all individuals in society. We assume that $X$ is a convex subset of some Euclidean space. One particular case is the classical setting of Anscombe and Aumann (1963) where $X$ is the set of lotteries over some finite set of prizes. Another is when $X$ is a convex subset of the set $\mathbb{R}^{n}$ of monetary allocations or, more generally, of the set $\mathbb{R}^{d n}$ of allocations of a finite number $d$ of commodities. Since $X$ is convex, given any two acts

\footnotetext{
${ }^{4}$ For instance, the common-taste unambiguous Pareto principle allows the policy maker to consider plausible all models that at least one individual considers plausible, as recently proposed by Brunnermeier et al. (2014).
} 
$f, g \in F$ and any coefficient $\lambda \in[0,1]$ there exists a "mixed" act $\lambda f+(1-\lambda) g \in F$ yielding outcome $\lambda f(s)+(1-\lambda) g(s)$ in each state $s \in S$.

\subsection{Unambiguous preferences}

Each individual $i=1, \ldots, n$ has preferences over the acts in $F$, described by a binary relation $\succsim_{i}$ on $F$. That is to say, we write $f \succsim_{i} g$ when individual $i$ weakly prefers act $f$ to act $g$. As usual we use $\succ_{i}$ to indicate strict preference and $\sim_{i}$ for indifference. Society also has preferences described by a binary relation $\succsim_{0}$ on $F$. We assume that all these relations are unambiguous preference relations in the following sense (we use the generic notation $\succsim$ when the subscript $i$ can be omitted).

Definition 1. A binary relation $\succsim$ on $F$ is an unambiguous preference relation if there exist a non-constant, affine utility function $u: X \rightarrow \mathbb{R}$ and a closed, convex set $P$ of probability distributions on $S$, such that for all acts $f, g \in F$,

$$
f \succsim g \text { if and only if } E_{p}(u(f)) \geq E_{p}(u(g)) \text { for all } p \in P
$$

where $E_{p}(u(f))=\sum_{s \in S} p(s) u(f(s))$ for all $f \in F$ and $p \in P$.

$P$ is interpreted as the set of all probability distributions (probabilistic models) the individual (or society) considers a plausible description of the uncertainty about the state of the world. When $P$ contains a single probability distribution, the agent has standard subjective expected utility (SEU) preferences and prefers the act yielding the highest expected utility under this probability distribution. When $P$ contains multiple probability distributions, the agent only has an unambiguous preference between two acts when one act yields a higher expected utility than the other under every distribution. If the act yielding the highest expected utility depends on which distribution in $P$ is used, then the individual has no unambiguous preference between the two acts. ${ }^{5}$

Unambiguous preferences were introduced by Bewley (2002). They satisfy all the properties characterizing SEU preferences, except the completeness property. The belief $P$ is uniquely pinned down by the preference relation $\succsim$, whereas the utility function $u$ is cardinally unique (i.e. unique up to a positive affine transformation).

\subsection{Taste heterogeneity}

We focus on situations where individuals' tastes or interests, as captured by their respective utility functions, are not perfectly aligned. More precisely, we shall assume that for each individual one can find two constant acts between which this individual is the only one to have a strict preference (all other individuals being indifferent). The profile $\left(\succsim_{i}\right)_{i=1}^{n}$ of individuals' unambiguous preference relations is said to satisfy $c$-diversity if for all $i=1, \ldots, n$, there exist $x, y \in X$ such that $x \succ_{i} y$ whereas $x \sim_{j} y$ for all $j=1, \ldots, n, j \neq i .^{6}$

\footnotetext{
${ }^{5}$ The individual may still come up with an overall preference judgment or reveal a behavioral disposition for one of the two acts; such a preference would simply not be unambiguous. See Section 3.

${ }^{6}$ This property, which is standard in the preference aggregation literature, is often named "independent prospects".
} 
C-diversity is known to be equivalent to the individuals' utility functions being linearly independent (note that this is only possible if $X$ is at least $n$-dimensional; Weymark, 1993). Thus individual tastes cannot be in full agreement, but neither can they be in full disagreement. In fact c-diversity implies that the profile $\left(\succsim_{i}\right)_{i=1}^{n}$ satisfies the following c-minimal agreement property: there exist two constant acts $x, y \in X$ such that $x \succ_{i} y$ for all $i=1, \ldots, n$.

\section{Robust Pareto principles}

This section contains the main results of the paper. We first state a robust version of the standard Pareto principle and characterize its implications for social preferences. We then consider a weakening of this robust principle to a particular subset of acts, yielding a more general characterization.

\subsection{Unambiguous Pareto dominance}

The following is the most straightforward application of the Pareto principle in our context. It simply states that if all individuals unambiguously prefer $f$ to $g$, then so should society.

Definition 2. The social unambiguous preference relation $\succsim_{0}$ satisfies unambiguous Pareto dominance with respect to the profile $\left(\succsim_{i}\right)_{i=1}^{n}$ of individual unambiguous preference relations if for all acts $f, g \in F, f \succsim_{0} g$ whenever $f \succsim_{i} g$ for all $i=1, \ldots, n$.

The following characterization result shows that the unambiguous Pareto principle provides guidance as to which beliefs society may adopt, provided individuals' beliefs are not too heterogeneous.

Theorem 1. Let $\succsim_{i}$ be an unambiguous preference relation on $F$ with representation $\left(u_{i}, P_{i}\right)$ for all $i=0, \ldots, n$. Assume $\left(\succsim_{i}\right)_{i=1}^{n}$ satisfies c-diversity. Then $\succsim_{0}$ satisfies unambiguous Pareto dominance with respect to $\left(\succsim_{i}\right)_{i=1}^{n}$ if and only if there exist a vector of weights $\theta \in \mathbb{R}_{+}^{n}, \theta \neq 0$, and a constant $\gamma \in \mathbb{R}$ such that

$$
u_{0}=\sum_{i=1}^{n} \theta_{i} u_{i}+\gamma \quad \text { and } \quad P_{0} \subseteq \bigcap_{\substack{i=1 \\ \theta_{i}>0}}^{n} P_{i}
$$

Theorem 1 provides a way of aggregating individuals' tastes and beliefs. The social utility function is a utilitarian - or linear - aggregation of individuals' utility functions. This simply comes from applying the unambiguous Pareto principle to the constant acts, where it reduces to the standard Pareto principle since beliefs do not matter for the evaluation of these acts. It is thus a direct extension of Harsanyi (1955)'s aggregation theorem.

More interesting is the way the social belief is constrained by individuals'. When individual beliefs are compatible in the sense of having a non-empty intersection, the social belief must lie inside this intersection. The unambiguous Pareto principle thus yields a strong but intuitive prescription: society must only use probability distributions that all individuals consider plausible. This in particular implies that society has a more precise belief than all the individuals. The condition that the intersection of individuals' beliefs is non-empty is not new in the literature; 
it appears for instance in Rigotti and Shannon (2005), where it is needed to prove that, absent any aggregate risk, the set of Pareto optima coincides with the set of full insurance allocations.

If individual beliefs are incompatible - or have an empty intersection - then some individuals have to be "excluded" as it were, i.e. given zero weight in the social utility function. For instance, if all individuals have distinct precise beliefs, then the only way for society to satisfy unambiguous Pareto dominance is that its preferences coincide with those of a particular individual, who then acts as a dictator. More generally, SEU individuals are either given zero weight or are dictators: any individual with SEU preferences and a non-zero weight forces society to have SEU preferences with her prior, in a way forcing her "certitude" on the society. ${ }^{7}$

\subsection{Common-taste unambiguous Pareto dominance}

When individuals have incompatible beliefs and society does not wish to exclude some of them, the unambiguous Pareto principle yields no prescription for society. To recover some guidance in these situations, we now restrict this principle to acts that are "consensual" in a particular sense.

Let us start with a situation where our notion of consensus takes a particularly simple form. Consider two constant acts $x, y \in X$ such that $x \succ_{i} y$ for all $i=1, \ldots, n$ (such acts exist by c-minimal agreement) and two acts $f, g \in F$ that never yield an outcome different from $x$ or $y$ in any state. Such acts are consensual in the sense that all individuals agree state by state on the ranking of their respective outcomes: for all $s \in S, f(s)$ is either unanimously "good" - if it is $x$ - or unanimously "bad" - if it is $y$ - and similarly for $g$. Now if $f$ unambiguously Pareto dominates $g$ then all individuals, notwithstanding their incompatible and potentially imprecise beliefs, further agree that $f$ is more likely than $g$ to yield the "good" outcome. Put differently, they would continue to unanimously prefer $f$ to $g$ if they agreed to "pool" their beliefs - each of them incorporating the others' beliefs into her own.

More generally, we say that two acts are "common-taste" acts if all individuals have the same cardinal preferences over their possible outcomes. ${ }^{8}$ Formally, given an act $f$, let $f(S)=$ $\{f(s): s \in S\}$ denote the image of $f$, i.e. the set of all possible outcomes of $f$. Given a set $Y$ of outcomes, let $\operatorname{conv}(Y)$ denote the convex hull of $Y$, i.e. the set of all convex combinations (or weighted averages) of outcomes in $Y$. Two acts $f$ and $g$ are common-taste acts if $x \succsim_{i} y$ is equivalent to $x \succsim_{j} y$ for all $x, y \in \operatorname{conv}(f(S) \cup g(S))$ and $i, j=1, \ldots, n$. Equivalently, $f$ and $g$ are common-taste acts if all individual utility functions, once restricted to the set of all possible outcomes of these two acts, are identical up to positive affine transformations.

Definition 3. The social unambiguous preference relation $\succsim_{0}$ satisfies common-taste unambiguous Pareto dominance with respect to the profile $\left(\succsim_{i}\right)_{i=1}^{n}$ of individual unambiguous preference relations if for all common-taste acts $f, g \in F, f \succsim_{0} g$ whenever $f \succsim_{i} g$ for all $i=1, \ldots, n$.

Theorem 2. Let $\succsim_{i}$ be an unambiguous preference relation on $F$ with representation $\left(u_{i}, P_{i}\right)$ for all $i=0, \ldots, n$. Assume $\left(\succsim_{i}\right)_{i=1}^{n}$ satisfies c-minimal agreement. Then $\succsim_{0}$ satisfies common-taste

\footnotetext{
${ }^{7}$ A similar pattern was experimentally observed by Baillon et al. (2012).

${ }^{8}$ In the two-outcome situations discussed above there is no distinction between ordinal and cardinal preferences. This is no longer true with more than two outcomes and requiring identical cardinal preferences turns out to provide the relevant notion of common-taste acts for our purposes.
} 
unambiguous Pareto dominance with respect to $\left(\succsim_{i}\right)_{i=1}^{n}$ if and only if there exist a vector of weights $\theta \in \mathbb{R}_{+}^{n}, \theta \neq 0$, and a constant $\gamma \in \mathbb{R}$ such that

$$
u_{0}=\sum_{i=1}^{n} \theta_{i} u_{i}+\gamma, \quad \text { and } \quad P_{0} \subseteq \operatorname{conv}\left(\bigcup_{i=1}^{n} P_{i}\right)
$$

The common-taste unambiguous Pareto principle thus allows aggregation of unambiguous preferences even with incompatible beliefs. As in Theorem 1, society can have SEU preferences even if all individuals have imprecise beliefs. The opposite case is now also possible: society can have imprecise beliefs even if all individuals have SEU preferences, in which case social belief imprecision results from individual belief heterogeneity. Although more permissive than Theorem 1 in the way society's beliefs could be related to individuals', this result always provides guidance for the construction of these beliefs.

Remark 1. We would obtain the same characterization if we strengthened the common-taste unambiguous Pareto principle by focusing on the "involved" individuals, in the spirit of Gilboa et al. (2014): individual $i$ is involved in the comparison between $f$ and $g$ if $f(s) \nsim_{i} g(s)$ for some $s \in S$. That is, we would now say, more generally, that $f$ and $g$ are common-taste acts if $x \succsim_{i} y$ is equivalent to $x \succsim_{j} y$ for all $x, y \in \operatorname{conv}(f(S) \cup g(S))$ and all individuals $i, j$ that are involved in $f$ and $g$. Equivalently, $f$ and $g$ are common-taste acts if all individual utility functions, once restricted to the set of all possible outcomes of these two acts, are either identical up to positive affine transformations or constant.

Remark 2. Unlike Theorem 1, Theorem 2 does not require individual preferences to satisfy c-diversity but only c-minimal agreement. It is therefore applicable to the particular case where all individuals have identical tastes.

\section{Social optima and social choice}

This section turns to the problem of choosing a socially optimal act among a given set of feasible acts. We provide results helping society to compute the set of optimal acts and make a further selection among them.

A feasible act is optimal if no other feasible act is strictly preferred to it. When society has a precise belief $p_{0}$, the socially optimal acts are thus simply those that maximize expected social utility under $p_{0}$. When society has an imprecise belief $P_{0}$, on the other hand, the set of socially optimal acts cannot be computed by maximizing a single function, reflecting the incompleteness of the social unambiguous preference relation. However, we show that maximizing expected social utility under each "interior" distribution in $P_{0}$ separately always yields a lower bound for - that is, a subset of - this set. Moreover, when the feasible set is convex, doing so under all "boundary" distributions in $P_{0}$ as well yields an upper bound for this set. Finally, when the feasible set is polyhedral - determined by a finite system of weak linear inequalities - the lower bound is actually an exact characterization of the set of social optima. ${ }^{9}$

\footnotetext{
${ }^{9}$ Aumann $(1962,1964)$ and Evren $(2014)$ prove similar "scalarization" results in different settings.
} 
Proposition 1. Let $\succsim$ be an unambiguous preference relation with representation $(u, P)$ and $G$ be a subset of $F$. Then any act maximizing $E_{p}(u(f))$ in $G$ for some relatively interior $p \in P$ is optimal for $\succsim$ in $G$. Conversely, if $G$ is convex then any optimal act for $\succsim$ in $G$ maximizes $E_{p}(u(f))$ in $G$ for some $p \in P$, and if $G$ is polyhedral then any optimal act for $\succsim$ in $G$ maximizes $E_{p}(u(f))$ in $G$ for some relatively interior $p \in P .{ }^{10}$

Once the socially optimal acts are identified, society may wish to select among them by "completing" the social unambiguous ranking in a consistent way rather than picking an act arbitrarily. Formally, we say that a binary relation $\succsim^{\prime}$ on $F$ is a completion of an unambiguous preference relation $\succsim$ on $F$ if (i) $\succsim^{\prime}$ is complete, (ii) $f \succsim g$ implies $f \succsim^{\prime} g$ for all $f, g \in F$, and (iii) $x \succ y$ implies $x \succ^{\prime} y$ for all $x, y \in X$. As we next show, virtually any consistent completion can be interpreted as evaluating the different acts with varying degrees of "caution" in the following sense.

Definition 4. A binary relation $\succsim^{\prime}$ on $F$ is a variable caution choice rule for an unambiguous preference relation $\succsim$ on $F$ with representation $(u, P)$ if there exists a function $\alpha: F \rightarrow[0,1]$ such that for all acts $f, g \in F$,

$$
f \succsim^{\prime} g \text { if and only if } V(f) \geq V(g)
$$

where $V(f)=\alpha(f) \min _{p \in P} E_{p}(u(f))+(1-\alpha(f)) \max _{p \in P} E_{p}(u(f))$ for all $f \in F$.

The coefficient $\alpha(f)$ is interpreted as the degree of caution with which act $f$ is evaluated. It is unique whenever the minimal and maximal expected utilities of $f$ do not coincide (otherwise it is irrelevant), and independent of $(u, P)$. The most cautious rule corresponds to $\alpha(f)=1$ for all $f \in F$ (Gilboa et al., 2010). It is akin to the precautionary principle, each act being evaluated by its minimal expected utility. The least cautious rule corresponds to $\alpha(f)=0$ for all $f \in F$. More generally, taking $\alpha$ constant corresponds to the Hurwicz (1951) "optimism-pessimism" criterion. Letting $\alpha$ vary with the act allows for more general rules. For instance, choosing a distribution $p^{\prime} \in P$ and taking $\alpha(f)=\frac{\max _{p \in P} E_{p}(u(f))-E_{p^{\prime}}(u(f))}{\max _{p \in P} E_{p}(u(f))-\min _{p \in P} E_{p}(u(f))}$ corresponds to a SEU rule.

Proposition 2. If a binary relation $\succsim^{\prime}$ on $F$ is a transitive, c-Archimedean completion of an unambiguous preference relation $\succsim$ on $F$ then it is a variable caution choice rule for ${ }^{11}$

Transitivity requires the completion to rank acts in a consistent way. The c-Archimedean property, on the other hand, is a mild continuity requirement. When these two requirements are met, selecting among socially optimal acts thus amounts to adopting a more or less cautious attitude towards social belief imprecision. The degree of social caution may depend on the act under consideration.

Remark 3. The converse of Proposition 2 does not hold: some variable caution choice rules or $\alpha$ functions - reverse some unambiguous rankings and hence are not completions of it. The converse holds, however, for all the particular cases discussed above. It holds, more generally, if

\footnotetext{
${ }^{10} \mathrm{~A}$ distribution $p \in P$ is relatively interior if for every distribution $q \in P$, there exist a distribution $r \in P$ and a coefficient $\lambda \in(0,1)$ such that $p=\lambda q+(1-\lambda) r$.

${ }^{11} \mathrm{~A}$ binary relation $\succsim^{\prime}$ on $F$ is $c$-Archimedean if for all $f \in F$ and $x, y \in X$ such that $x \succ^{\prime} f \succ^{\prime} y$, there exist $\lambda, \mu \in(0,1)$ such that $\lambda x+(1-\lambda) y \succ^{\prime} f \succ^{\prime} \mu x+(1-\mu) y$.
} 
the definition of a variable caution choice rule is strengthened to further require that $V(f) \geq V(g)$ whenever $E_{p}(u(f)) \geq E_{p}(u(g))$ for all $p \in P$.

Remark 4. Our definition of a variable caution choice rule is identical to Cerreia-Vioglio et al. (2011)'s definition of a "generalized Hurwicz representation", except that they require $\succsim$ to be derived from $\succsim^{\prime}$ in a specific way whereas we more generally allow $\succsim$ to be any unambiguous preference relation admitting $\succsim^{\prime}$ as a completion. Cerreia-Vioglio et al. (2011) show that any "monotonic Bernoullian Archimedean" (MBA) preference relation admits such a representation. ${ }^{12}$ Any MBA preference relation is a transitive, c-Archimedean completion of some unambiguous preference relation, but the converse is not true as MBA preferences satisfy a stronger Archimedean property.

\section{Discussion and related literature}

In this section, we further discuss the relationship between our main results and the existing literature on social decisions. Whereas most of the literature assumes SEU preferences, a recent strand of papers considers ambiguity-sensitive- or non-expected utility - preferences.

\subsection{Social decisions with SEU preferences}

When individuals and society have SEU preferences and individual tastes are heterogeneous, respecting Pareto dominance is impossible unless all individuals (with non-zero weight) have identical beliefs (Hylland and Zeckhauser, 1979; Mongin, 1995, 1998, 2014). Theorem 1 generalizes this result to unambiguous preferences. This generalization is partly a possibility result: the unambiguous Pareto principle can accommodate simultaneous heterogeneity in tastes and beliefs, as long as beliefs are compatible. In the particular case where all individuals have SEU preferences, it also yields the following corollary, showing that the assumption that society has a precise belief is not necessary for the impossibility result.

Corollary 1. Let $\succsim_{0}$ be an unambiguous preference relation on $F$ and $\succsim_{i}$ be a SEU preference relation on $F$ for all $i=1, \ldots, n$. Assume $\left(\succsim_{i}\right)_{i=1}^{n}$ satisfies c-diversity. If $\succsim_{0}$ satisfies unambiguous Pareto dominance with respect to $\left(\succsim_{i}\right)_{i=1}^{n}$ then $\succsim_{0}$ is a SEU preference relation.

Gilboa et al. (2004) restrict the Pareto principle to "common-belief" acts, i.e. acts whose outcome only depends on events to which all individuals assign the same probability. In the setting of Savage (1954), they show that this restriction allows aggregation of SEU preferences with heterogeneous tastes and beliefs, and requires the social belief to be a weighted average of the individuals'. In an Anscombe-Aumann setting, Qu (2015) obtains the same characterization by restricting the Pareto principle to common-taste acts. These two restrictions have the same flavor of allowing society to ignore "spurious" unanimities (that is, cases where individuals agree for opposite reasons), which are the source of the impossibility. The Savage setting features a rich set of states, making the common-belief restriction stronger, whereas the Anscombe-Aumann

\footnotetext{
${ }^{12}$ The MBA class includes virtually all popular ambiguity models, such as maxmin expected utility (MEU; Gilboa and Schmeidler, 1989), Choquet expected utility (CEU; Schmeidler, 1989), smooth ambiguity (Klibanoff et al., 2005), variational (Maccheroni et al., 2006), and multiplier (Hansen and Sargent, 2001) preferences.
} 
setting features a rich set of outcomes, making the common-taste restriction stronger. Theorem 2 generalizes $\mathrm{Qu}$ (2015)'s result to unambiguous preferences. ${ }^{13}$

Gilboa et al. (2014) say that an act $f$ no-betting Pareto dominates an act $g$ if $f$ Pareto dominates $g$ and there exists a probability distribution $p$ on $S$ such that $E_{p}\left(u_{i}(f)\right) \geq E_{p}\left(u_{i}(g)\right)$ for every involved individual $i$ (their definition requires strict inequality, but this weak version is more directly comparable to ours). Their definition can be generalized to unambiguous preferences by requiring unambiguous Pareto dominance instead of Pareto dominance. Gayer et al. (2014) say that an act $f$ unanimity Pareto dominates an act $g$ if $E_{p_{j}}\left(u_{i}(f)\right) \geq E_{p_{j}}\left(u_{i}(g)\right)$ for all involved individuals $i, j$. Their definition (which again requires strict inequality) can also be generalized to unambiguous preferences by requiring that $E_{p_{j}}\left(u_{i}(f)\right) \geq E_{p_{j}}\left(u_{i}(g)\right)$ for all individuals $i, j$ and all probability distributions $p_{j} \in P_{j}$. Common-taste unambiguous Pareto dominance then implies unanimity unambiguous Pareto dominance, which itself implies no-betting unambiguous Pareto dominance and in turn unambiguous Pareto dominance. Moreover, the last two are equivalent when individual beliefs are compatible. Finally, Theorem 2 implies that it is equivalent for a social unambiguous preference relation to respect either one of the first two when c-minimal agreement holds.

Brunnermeier et al. (2014) propose a belief neutral social welfare criterion that essentially consists in a social unambiguous ranking whose belief is the convex hull of the individuals'. This corresponds to the particular case of Theorem 2 where individuals have SEU preferences and society has the least complete unambiguous preferences satisfying common-taste unambiguous Pareto dominance. The common-taste unambiguous Pareto principle thus provides foundations for a generalization of their criterion allowing, on the one hand, for more precise social beliefs or more complete social preferences - and, on the other hand, for imprecise individual beliefs.

\subsection{Social decisions with ambiguity-sensitive preferences}

When individuals and society have ambiguity-sensitive preferences and individual tastes are heterogeneous, respecting Pareto dominance becomes impossible even when all individuals have identical beliefs. This has been shown in various settings covering in particular the class of MBA preferences (Gajdos et al., 2008; Herzberg, 2013; Chambers and Hayashi, 2014; Mongin and Pivato, 2015; Zuber, 2015). In contrast, Theorems 1 and 2 show that unambiguous preferences allow the aggregation of imprecise beliefs.

Moreover, our results can be used to obtain positive aggregation results for ambiguitysensitive preferences as well. Indeed, an ambiguity-sensitive preference relation naturally induces a "revealed unambiguous preference" relation, capturing the part of the preference ranking that is not affected by the ambiguity the individual perceives (Ghirardato et al., 2004; Nehring, 2007; Klibanoff et al., 2014). For a MBA preference relation $\succsim$, this revealed unambiguous preference relation $\succsim^{*}$ is an unambiguous preference relation in the sense of Definition 1 , and $\succsim$ is a variable caution choice rule for $\succsim^{*}$. $\succsim$ can therefore be represented by a triple $(u, P, \alpha)$ where $(u, P)$ is

\footnotetext{
${ }^{13}$ Note that $\mathrm{Qu}(2015)$ also defines common-taste acts more narrowly as those yielding only convex combinations of two exogenously fixed outcomes between which individuals have a unanimous strict preference. Our more general definition yields the same characterization while retaining a stronger Pareto principle. See also Billot and Vergopoulos (2014) for a different resolution of the impossibility through an extension of the state space.
} 
as in Definition 1 and $\alpha$ is as in Definition $4 .{ }^{14}$ The function $\alpha$ is then interpreted as reflecting the individual's attitude towards the ambiguity she perceives.

We may therefore restrict the Pareto principle as follows: say that the social preference relation $\succsim_{0}$ satisfies revealed unambiguous Pareto dominance with respect to the profile $\left(\succsim_{i}\right.$ )$_{i=1}^{n}$ of individual preference relations if the social revealed unambiguous preference relation $\succsim_{0}^{*}$ satisfies unambiguous Pareto dominance with respect to the profile $\left(\succsim_{i}^{*}\right)_{i=1}^{n}$ of individual revealed unambiguous preference relations. This principle, and its restriction to common-taste acts, are then characterized as in Theorems 1 and 2, respectively. Note that these characterizations do not involve the functions $\alpha_{i}$ and thus relate the individuals' and society's beliefs independently of their ambiguity attitudes. We explicitly state the latter result.

Corollary 2. Let $\succsim_{i}$ be a MBA preference relation on $F$ with representation $\left(u_{i}, P_{i}, \alpha_{i}\right)$ for all $i=0, \ldots, n$. Assume $\left(\succsim_{i}\right)_{i=1}^{n}$ satisfies c-minimal agreement. Then $\succsim_{0}$ satisfies common-taste revealed unambiguous Pareto dominance with respect to $\left(\succsim_{i}\right)_{i=1}^{n}$ if and only if there exist a vector of weights $\theta \in \mathbb{R}_{+}^{n}, \theta \neq 0$, and a constant $\gamma \in \mathbb{R}$ such that

$$
u_{0}=\sum_{i=1}^{n} \theta_{i} u_{i}+\gamma \quad \text { and } \quad P_{0} \subseteq \operatorname{conv}\left(\bigcup_{i=1}^{n} P_{i}\right) .
$$

Several particular specifications of this general characterization have been studied within various subclasses of MBA preferences. Crès et al. (2011), Nascimento (2012), Hill (2012), and Gajdos and Vergnaud (2013) assume that individuals have identical tastes. Allowing for taste heterogeneity, $\mathrm{Qu}(2015)$ characterizes a strengthening of the common-taste Pareto principle within the MEU and CEU classes. Alon and Gayer (2015) assume that individuals have SEU preferences whereas society has MEU preferences and characterize the unanimity Pareto principle.

\footnotetext{
${ }^{14}$ Note that in this approach $\succsim$ is the only primitive relation whereas $\succsim^{*}$ is derived from $\succsim$. Note also that the definition of $\succsim^{*}$ - or, equivalently, of $P$ - by Ghirardato et al. (2004) and Nehring (2007) does not necessarily coincide with that by Klibanoff et al. (2014), the former being generally more complete.
} 


\section{Appendix: proofs}

Given a utility function $u: X \rightarrow \mathbb{R}$ and a probability distribution $p$ on $S$, define the "statedependent utility" function $w_{u, p}: X \times S \rightarrow \mathbb{R}$ by $w_{u, p}(x, s)=p(s) u(x)$. Given a set $P$ of probability distributions on $S$, let $W_{u, P}=\left\{w_{u, p}: p \in P\right\}$. Let

$$
C=\left\{c \in \mathbb{R}^{X \times S}: c(x, s)=c(y, s) \text { for all } x, y \in X \text { and } s \in S\right\}
$$

denote the set of "state-dependent constant" functions. Let cone(.) denote conic hull.

Lemma 1. Let $\succsim_{i}$ be an unambiguous preference relation on $F$ with representation $\left(u_{i}, P_{i}\right)$ for all $i=0, \ldots, n$. Assume $\left(\succsim_{i}\right)_{i=1}^{n}$ satisfies c-minimal agreement. Then $\succsim_{0}$ satisfies unambiguous Pareto dominance with respect to $\left(\succsim_{i}\right)_{i=1}^{n}$ if and only if

$$
W_{u_{0}, P_{0}} \subseteq \sum_{i=1}^{n} \operatorname{cone}\left(W_{u_{i}, P_{i}}\right)+C .
$$

Proof. $X$ has a finite affine basis $\hat{X}$ since it is a subset of a Euclidean space. Given a statedependent utility function $w: X \times S \rightarrow \mathbb{R}$, denote by $\hat{w}$ its restriction to $\hat{X} \times S$. Given a set $W$ of such functions, denote by $\hat{W}$ the set of corresponding restrictions. Then (1) is equivalent to

$$
\hat{W}_{u_{0}, P_{0}} \subseteq \sum_{i=1}^{n} \operatorname{cone}\left(\hat{W}_{u_{i}, P_{i}}\right)+\hat{C} .
$$

It follows from a straightforward generalization of Danan et al. (2015)'s aggregation theorem that $\succsim_{0}$ satisfies unambiguous Pareto dominance with respect to $\left(\succsim_{i}\right)_{i=1}^{n}$ if and only if $W_{u_{0}, P_{0}}$ is included in the closure of the right hand side of (2). Hence it suffices to prove that this right hand side is closed. We first show that cone $\left(\hat{W}_{u_{i}, P_{i}}\right)+\hat{C}$ is a closed, convex cone for all $i=1, \ldots, n$. That it is a convex cone is easily checked. For closedness, note that $0 \notin \hat{W}_{u_{i}, P_{i}}$ since $u_{i}$ is nonconstant and, hence, $\operatorname{cone}\left(\hat{W}_{u_{i}, P_{i}}\right)$ is closed since $\hat{W}_{u_{i}, P_{i}}$ is compact and convex (Rockafellar, 1970, Corollary 9.6.1). Moreover, cone $\left(\hat{W}_{u_{i}, P_{i}}\right) \cap \hat{C}=\{0\}$ and, hence, cone $\left(\hat{W}_{u_{i}, P_{i}}\right)+\hat{C}$ is closed as well (Rockafellar, 1970, Corollary 9.1.3).

It remains to show that the sum of these closed, convex cones is itself closed. As explained in Danan et al. (2015), this will be the case if there exist two acts $f, g \in F$ such that, for all $i=1, \ldots, n$ and all $g_{i} \in F$ such that $f \succsim_{i} g_{i}$, there exist $g_{i}^{\prime} \in F$ and $\lambda_{i} \in(0,1)$ such that $f \succsim_{i} g_{i}^{\prime}$ and $g=\lambda_{i} g_{i}+\left(1-\lambda_{i}\right) g_{i}^{\prime}$. To establish this property, recall that by c-minimal agreement, there exist $x, y \in X$ such that $x \succ_{i} y$ for all $i=1 \ldots, n$. Hence, for all $i=1, \ldots, n$, there exists an open neighborhood $Y_{i}$ of $y$ in $X$ such that $x \succ_{i} z$ for all $z \in Y_{i}$. Let $g_{i} \in F$ such that $x \succsim_{i} g_{i}$ and, given a coefficient $\lambda \in(0,1)$, let $g_{i}^{\prime}=\frac{1}{1-\lambda} y-\frac{\lambda}{1-\lambda} g_{i}$. Then $y=\lambda g_{i}+(1-\lambda) g_{i}^{\prime}$. Moreover, since $S$ is finite, there exists $\lambda \in(0,1)$ small enough so that $g_{i}^{\prime} \in Y^{S} \subset F$ and, hence, $x \succ_{i} g_{i}^{\prime}(s)$ for all $s \in S$. It follows that $x \succsim_{i} g_{i}^{\prime}$.

Proof of Theorem 1. The "if" part is easily checked. For the "only if" part, assume $\succsim_{0}$ satisfies unambiguous Pareto dominance with respect to $\left(\succsim_{i}\right)_{i=1}^{n}$. Restricting attention to the constant acts, unambiguous Pareto dominance reduces to standard Pareto dominance. We can therefore 
apply Harsanyi (1955)'s aggregation theorem (for a rigorous proof in our setting, see de Meyer and Mongin, 1995) to obtain $\theta \in \mathbb{R}_{+}^{n}$ and $\gamma \in \mathbb{R}$ such that $u_{0}=\sum_{i=1}^{n} \theta_{i} u_{i}+\gamma . \theta$ and $\gamma$ are unique by c-diversity. Moreover, $\theta \neq 0$ since $u_{0}$ is non-constant.

It remains to prove that for all $p_{0} \in P_{0}$ and all $i=1, \ldots, n$ such that $\theta_{i}>0, p_{0} \in P_{i}$. To this end, note that by Lemma 1 , there exist $\left(p_{i}\right)_{i=1}^{n} \in \prod_{i=1}^{n} P_{i}, \theta^{\prime} \in \mathbb{R}_{+}^{n}$, and $c^{\prime} \in C$ such that

$$
w_{u_{0}, p_{0}}=\sum_{i=1}^{n} \theta_{i}^{\prime} w_{u_{i}, p_{i}}+c^{\prime}
$$

It follows that

$$
p_{0}(s) u_{0}(x)=\sum_{i=1}^{n} \theta_{i}^{\prime} p_{i}(s) u_{i}(x)+c^{\prime}(s)
$$

for all $s \in S$ and $x \in X$, where $c^{\prime}(s)$ stands for $c^{\prime}(x, s)$ since the latter is independent of $x$. Summing over $S$ yields

$$
u_{0}(x)=\sum_{i=1}^{n} \theta_{i}^{\prime} u_{i}(x)+\sum_{s \in S} c^{\prime}(s)
$$

for all $x \in X$, so that $\theta=\theta^{\prime}$ and $\gamma=\sum_{s \in S} c^{\prime}(s)$. Hence (3) implies that

$$
p_{0}(s)\left(u_{0}(x)-u_{0}(y)\right)=\sum_{i=1}^{n} \theta_{i} p_{i}(s)\left(u_{i}(x)-u_{i}(y)\right)
$$

and, hence, that

$$
\sum_{i=1}^{n} \theta_{i}\left(p_{0}(s)-p_{i}(s)\right)\left(u_{i}(x)-u_{i}(y)\right)=0
$$

for all $s \in S$ and $x, y \in X$. Fix an individual $i$ such that $\theta_{i}>0$. By c-diversity, there exist $x, y \in X$ such that $u_{i}(x)>u_{i}(y)$ whereas $u_{j}(x)=u_{j}(y)$ for all $j=1, \ldots, n, j \neq i$. By (4), it follows that $p_{0}(s)=p_{i}(s)$ for all $s \in S$, so that $p_{0}=p_{i} \in P_{i}$.

Proof of Theorem 2. The "if" part is easily checked. For the "only if" part, assume $\succsim_{0}$ satisfies common-taste unambiguous Pareto dominance with respect to $\left(\succsim_{i}\right)_{i=1}^{n}$. As in the proof of Theorem 1 , we first restrict attention to the constant acts to obtain $\theta \in \mathbb{R}_{+}^{n}, \theta \neq 0$, and $\gamma \in \mathbb{R}$ such that $u_{0}=\sum_{i=1}^{n} \theta_{i} u_{i}+\gamma$.

It remains to prove that for all $p_{0} \in P_{0}$, there exist $\left(p_{i}\right)_{i=1}^{n} \in \prod_{i=1}^{n} P_{i}$ and $\lambda \in \Delta^{n}$ such that $p_{0}=\sum_{i=1}^{n} \lambda_{i} p_{i}$. By c-minimal agreement, there exist $x, y \in X$ such that $x \succ_{i} y$ for all $i=1, \ldots, n$. Hence all acts in $\operatorname{conv}(\{x, y\})^{S}$ are common-taste acts. It follows that $x \succ_{0} y$, so that individual and social preferences all agree on $\operatorname{conv}(\{x, y\})$. Hence for all $i=1, \ldots, n$, there exist $a_{i} \in \mathbb{R}_{+}, a_{i}>0$, and $b_{i} \in \mathbb{R}$ such that

$$
u_{i}(z)=a_{i} u_{0}(z)+b_{i}
$$

for all $z \in \operatorname{conv}(\{x, y\})$. We can therefore use the common-taste unambiguous Pareto principle 
to show, as in the proof of Theorem 1 , that for all $p_{0} \in P_{0}$, there exist $\left(p_{i}\right)_{i=1}^{n} \in \prod_{i=1}^{n} P_{i}, \theta^{\prime} \in \mathbb{R}_{+}^{n}$, and $c^{\prime} \in C$ such that

$$
p_{0}(s) u_{0}(z)=\sum_{i=1}^{n} \theta_{i}^{\prime} p_{i}(s) u_{i}(z)+c^{\prime}(s)
$$

for all $s \in S$ and $z \in \operatorname{conv}(\{x, y\})$. Summing over $S$ and using (5) yields

$$
u_{0}(z)=\sum_{i=1}^{n} \theta_{i}^{\prime} u_{i}(z)+\sum_{s \in S} c^{\prime}(s)=\sum_{i=1}^{n} \theta_{i}^{\prime} a_{i} u_{0}(z)+\sum_{i=1}^{n} \theta_{i}^{\prime} b_{i}+\sum_{s \in S} c^{\prime}(s)
$$

for all $z \in \operatorname{conv}(\{x, y\})$, so that $\sum_{i=1}^{n} \theta_{i}^{\prime} a_{i}=1$ and $\sum_{i=1}^{n} \theta_{i}^{\prime} b_{i}=-\sum_{s \in S} c^{\prime}(s)$ since $u_{0}$ is nonconstant on $\operatorname{conv}(\{x, y\})$. Hence (6) implies that

$$
p_{0}(s)\left(u_{0}(x)-u_{0}(y)\right)=\sum_{i=1}^{n} \theta_{i}^{\prime} p_{i}(s)\left(u_{i}(x)-u_{i}(y)\right)=\sum_{i=1}^{n} \theta_{i}^{\prime} p_{i}(s) a_{i}\left(u_{0}(x)-u_{0}(y)\right)
$$

and, hence, that

$$
p_{0}(s)=\sum_{i=1}^{n} \theta_{i}^{\prime} a_{i} p_{i}(s)
$$

for all $s \in S$, so that $p_{0}=\sum_{i=1}^{n} \theta_{i}^{\prime} a_{i} p_{i}$. Let $\lambda=\left(\theta_{i}^{\prime} a_{i}\right)_{i=1}^{n} \in \mathbb{R}^{n}$. Since $\theta_{i}^{\prime} \geq 0$ and $a_{i}>0$ for all $i=1, \ldots, n$ and $\sum_{i=1}^{n} \theta_{i}^{\prime} a_{i}=1$, we have $\lambda \in \Delta^{n}$.

Proof of Proposition 1. First, let $G$ be any subset of $X$ and let $g \in \arg \max _{f \in G} E_{p}(u(f))$ for some relatively interior $p \in P$. We show that $g$ is optimal for $\succsim$ in $G$. Suppose not, i.e. there exists $f \in G$ such that $f \succ g$. It must then be that $E_{p}(u(f))=E_{p}(u(g))$ whereas $E_{q}(u(f))>E_{q}(u(g))$ for some $q \in P$. Moreover, since $p$ is relatively interior in $P$, there exist $r \in P$ and $\lambda \in(0,1)$ such that $p=\lambda q+(1-\lambda) r$, i.e. $r=\frac{1}{1-\lambda} p-\frac{\lambda}{1-\lambda} q$. It follows that

$$
E_{r}(u(f))=\frac{1}{1-\lambda} E_{p}(u(f))-\frac{\lambda}{1-\lambda} E_{q}(u(f))<\frac{1}{1-\lambda} E_{p}(u(g))-\frac{\lambda}{1-\lambda} E_{q}(u(g))=E_{r}(u(g)),
$$

contradicting $f \succ g$.

Next, assume $G$ is convex and $g \in G$ is optimal for $\succsim$ in $G$, i.e. there exists no $f \in G$ such that $f \succ g$. We show that $g \in \arg \max _{f \in G} E_{p}(u(f))$ for some $p \in P$. Let

$$
\begin{aligned}
& A=\left\{v \in \mathbb{R}^{S}: \text { there exists } f \in G \text { such that } v(s)=u(f(s))-u(g(s)) \text { for all } s \in S\right\}, \\
& B=\left\{v \in \mathbb{R}^{S}: E_{q}(v)>0 \text { for all } q \in P\right\} .
\end{aligned}
$$

Then $A$ is convex since $G$ is convex and $u$ is affine, and $B$ is a convex cone whose dual cone is cone $(P)$. Moreover, since $g$ is optimal for $\succsim$ in $G, A$ and $B$ must be disjoint by Definition 1 . Hence by a separation argument (Rockafellar, 1970, Theorem 11.3), there exists $p \in \mathbb{R}^{S}, p \neq 0$, 
such that

$$
\sum_{s \in S} p(s) b(s) \geq 0 \geq \sum_{s \in S} p(s) a(s)
$$

for all $a \in A$ and $b \in B$. The former inequality implies that $p \in \operatorname{cone}(P)$, so we can assume without loss of generality that $p \in P$. The latter inequality then implies that $E_{p}(u(g)) \geq$ $E_{p}(u(f))$ for all $f \in G$.

Finally, assume $G$ is polyhedral and $g \in G$ is optimal for $\succsim$ in $G$, i.e. there exists no $f \in G$ such that $f \succ g$. We show that $g \in \arg \max _{f \in G} E_{p}(u(f))$ for some relatively interior $p \in P$. Define $A$ as above and let

$$
B^{\prime}=\left\{v \in \mathbb{R}^{S}: E_{q}(v) \geq 0 \text { for all } q \in P\right\}
$$

Then $A$ is polyhedral since $G$ is polyhedral and $u$ is affine, and $B^{\prime}$ is a closed, convex cone whose dual cone is cone $(P)$. Since $A$ is polyhedral and $0 \in A$, cone $(A)$ is a closed, convex cone (Rockafellar, 1970, Corollary 19.7.1). We also have $B^{\prime}=B_{1}^{\prime}+B_{2}^{\prime}$ where $B_{1}^{\prime}$ is the lineality space of $B^{\prime}$ and $B_{2}^{\prime}$ is a pointed, closed, convex cone orthogonal to $B_{1}^{\prime}$. Since $B_{2}^{\prime}$ is pointed, there exists a compact, convex set $D \subset B_{2}^{\prime}, 0 \notin D$, such that cone $(D)=B_{2}^{\prime}$ and, hence, $\operatorname{cone}\left(B_{1}^{\prime}+D\right)=B^{\prime}$. Moreover, since $g$ is optimal for $\succsim$ in $G$, we have $A \cap B^{\prime} \subseteq B_{1}^{\prime}$ by Definition 1 and, hence, $A$ and $B_{1}^{\prime}+D$ must be disjoint since $0 \notin D$. Hence by a separation argument (Rockafellar, 1970, Corollary 20.3.1), there exists $q \in \mathbb{R}^{S}, q \neq 0$, and $\varepsilon \in \mathbb{R}$ such that

$$
\sum_{s \in S} q(s) b(s)>\varepsilon \geq 0 \geq \sum_{s \in S} q(s) a(s)
$$

for all $a \in A$ and $b \in B_{1}^{\prime}+D$. It follows that there exists an open neighborhood $Q$ of $q$ such that, for all $r \in Q$,

$$
\sum_{s \in S} r(s) b(s) \geq 0 \geq \sum_{s \in S} r(s) a(s)
$$

for all $a \in A$ and $b \in B^{\prime}$. The former inequality implies that $Q \subset \operatorname{cone}(P)$, so we can assume without loss of generality that $q \in P$. By definition, $Q$ must then contain a relatively interior $p \in P$. The latter inequality then implies that $E_{p}(u(g)) \geq E_{p}(u(f))$ for all $f \in G$.

Proof of Proposition 2. Assume $\succsim^{\prime}$ is a transitive, c-Archimedean completion of an unambiguous preference relation $\succsim$ on $F$ with representation $(u, P)$. First note that since $\succsim^{\prime}$ is a completion of $\succsim$ and by Definition 1 , we have

$$
x \succsim^{\prime} y \text { if and only if } x \succsim y \text { if and only if } u(x) \geq u(y)
$$

for all $x, y \in X$. For all $f \in F$, let $x_{f} \in \arg \max _{s \in S} u(f(s))$ and $y_{f} \in \arg \min _{s \in S} u(f(s))$. This is well-defined since $S$ is finite. We then have

$$
u\left(x_{f}\right) \geq \max _{p \in P} E_{p}(u(f)) \geq \min _{p \in P} E_{p}(u(f)) \geq u\left(y_{f}\right) .
$$


Since $X$ is convex and $u$ is affine, there then exist $x_{f}^{\prime}, y_{f}^{\prime} \in \operatorname{conv}\left(\left\{x_{f}, y_{f}\right\}\right)$ such that $u\left(x_{f}^{\prime}\right)=$ $\max _{p \in P} E_{p}(u(f))$ and $u\left(y_{f}^{\prime}\right)=\min _{p \in P} E_{p}(u(f))$. It follows that $x_{f}^{\prime} \succsim f \succsim y_{f}^{\prime}$ by Definition 1 and, hence, $x_{f}^{\prime} \succsim^{\prime} f \succsim^{\prime} y_{f}^{\prime}$ since $\succsim^{\prime}$ is a completion of $\succsim$.

We now show that there exist $\alpha(f) \in[0,1]$ such that

$$
f \sim^{\prime} \alpha(f) y_{f}^{\prime}+(1-\alpha(f)) x_{f}^{\prime}
$$

If $f \sim^{\prime} x_{f}^{\prime}$ or $f \sim^{\prime} y_{f}^{\prime}$ then we are done, so assume $x_{f}^{\prime} \succ^{\prime} f \succ^{\prime} y_{f}^{\prime}$. By (7), we then have $u\left(x_{f}^{\prime}\right)>u\left(y_{f}^{\prime}\right)$. Let

$$
\begin{aligned}
L & =\left\{\lambda \in[0,1]: \lambda y_{f}^{\prime}+(1-\lambda) x_{f}^{\prime} \succsim^{\prime} f\right\} \\
M & =\left\{\mu \in[0,1]: f \succsim^{\prime} \mu y_{f}^{\prime}+(1-\mu) x_{f}^{\prime}\right\} .
\end{aligned}
$$

We then have $L \cup M=[0,1]$ since $\succsim^{\prime}$ is complete. Moreover, for all $\lambda \in L$ and $\mu \in M$, we have $\lambda y_{f}^{\prime}+(1-\lambda) x_{f}^{\prime} \succsim^{\prime} \mu y_{f}^{\prime}+(1-\mu) x_{f}^{\prime}$ since $\succsim^{\prime}$ is transitive and, hence, $u\left(\lambda y_{f}^{\prime}+(1-\lambda) x_{f}^{\prime}\right) \geq$ $u\left(\mu y_{f}^{\prime}+(1-\mu) x_{f}^{\prime}\right)$ by (7). Since $u$ is affine and $u\left(x_{f}^{\prime}\right)>u\left(y_{f}^{\prime}\right)$, this is only possible if $\lambda \leq \mu$. It follows that $\sup L=\inf M$. Finally, $L$ and $M$ are closed since $\succsim^{\prime}$ is c-Archimedean and, hence, $\sup L=\max L$ and $\inf M=\min M$. Hence, letting $\alpha(f)=\max L=\min M$ and $z_{f}=\alpha(f) y_{f}^{\prime}+(1-\alpha(f)) x_{f}^{\prime}$, we have $f \sim^{\prime} z_{f}$.

Finally, let $V(f)=u\left(z_{f}\right)$. Since $u$ is affine, we then have

$$
\left.V(f)=\alpha(f) u\left(y_{f}^{\prime}\right)+(1-\alpha(f)) u\left(x_{f}^{\prime}\right)=\alpha(f) \min _{p \in P} E_{p} u(f)\right)+(1-\alpha(f)) \max _{p \in P} E_{p}(u(f)) .
$$

Moreover, since $\succsim^{\prime}$ is transitive and by (7), we have

$$
f \succsim^{\prime} g \text { if and only if } z_{f} \succsim^{\prime} z_{g} \text { if and only if } V(f) \geq V(g)
$$

for all acts $f, g \in F$, which completes the proof.

\section{References}

Alon, S. And G. Gayer (2015): "Utilitarian Preferences with Multiple Priors," Mimeo.

Anscombe, F. J. And R. J. Aumann (1963): "A Definition of Subjective Probability," The Annals of Mathematical Statistics, 34, 199-205.

Aumann, R. J. (1962): "Utility theory without the completeness axiom," Econometrica, 30, $445-462$.

(1964): "Utility theory without the completeness axiom: A correction," Econometrica, $32,210-212$.

Baillon, A., L. Cabantous, And P. P. Wakker (2012): "Aggregating imprecise or conflicting beliefs: An experimental investigation using modern ambiguity theories," Journal of Risk and Uncertainty, 44, 115-147. 
Bewley, T. F. (2002): "Knightian decision theory. Part I," Decisions in Economics and Finance, $25,79-110$.

Billot, A. And V. Vergopoulos (2014): "Utilitarianism with Prior Heterogeneity," Mimeo.

Brunnermeier, M. K., A. Simsek, And W. Xiong (2014): "A Welfare Criterion for Models with Distorted Beliefs," Quarterly Journal of Economics, 129, 1753-1797.

Cerreia-Vioglio, S., P. Ghirardato, F. Maccheroni, M. Marinacci, and M. SinisCALCHI (2011): "Rational preferences under ambiguity," Economic Theory, 48, 341-375.

Chambers, C. P. And T. Hayashi (2014): "Preference aggregation with incomplete information," Econometrica, 82, 589-599.

Crès, H., I. GilboA, AND N. Vieille (2011): "Aggregation of multiple prior opinions," Journal of Economic Theory, 146, 2563-2582.

Danan, E., T. Gajdos, And J.-M. Tallon (2015): "Harsanyi's Aggregation Theorem with Incomplete Preferences," American Economic Journal: Microeconomics, 7, 61-69.

De Meyer, B. And P. Mongin (1995): "A note on affine aggregation," Economics Letters, 47, $177-183$.

Evren, O. (2014): "Scalarization methods and expected multi-utility representations," Journal of Economic Theory, 151, 30-63.

Gajdos, T., J.-M. Tallon, And J.-C. Vergnaud (2008): "Representation and aggregation of preferences under uncertainty," Journal of Economic Theory, 141, 68-99.

Gajdos, T. And J.-C. Vergnaud (2013): "Decisions with conflicting and imprecise information," Social Choice and Welfare, 41, 427-452.

Gayer, G., I. Gilboa, L. Samuelson, And D. Schmeidler (2014): "Pareto Efficiency with Different Beliefs," Journal of Legal Studies, 43, S151-S171.

Ghirardato, P., F. Maccheroni, and M. Marinacci (2004): "Differentiating ambiguity and ambiguity attitude," Journal of Economic Theory, 118, 133-173.

Gilboa, I., F. Maccheroni, M. Marinacci, and D. Schmeidler (2010): "Objective and Subjective Rationality in a Multiple Prior Model," Econometrica, 78, 755-770.

Gilboa, I., D. Samet, And D. Schmeidler (2004): "Utilitarian Aggregation of Beliefs and Tastes," Journal of Political Economy, 112, pp. 932-938.

Gilboa, I., L. Samuelson, And D. Schmeidler (2014): "No-Betting-Pareto Dominance," Econometrica, 82, 1405-1442.

GilboA, I. And D. Schmeidler (1989): "Maxmin expected utility with non-unique prior," Journal of Mathematical Economics, 18, 141-153. 
Hansen, L. P. And T. J. Sargent (2001): "Robust Control and Model Uncertainty," American Economic Review, 91, 60-66.

(2008): Robustness, Princeton University Press.

Harsanyi, J. C. (1955): "Cardinal Welfare, Individualistic Ethics, and Interpersonal Comparisons of Utility," Journal of Political Economy, 63, 309-321.

Henry, C. (2006): "Decision Making under Scientific, Political and Economic Uncertainty," Mimeo.

HerzBerg, F. (2013): "Aggregation of Monotonic Bernoullian Archimedean preferences: Arrovian impossibility results," Center for Mathematical Economics Working Papers, 488, Bielefeld University.

HiLl, B. (2012): "Unanimity and the aggregation of multiple prior opinions," Mimeo.

Hurwicz, L. (1951): "Optimality Criteria for Decision Making under Ignorance," Cowles Commission Discussion Papers: Statistics, 370.

Hylland, A. And R. Zeckhauser (1979): "The Impossibility of Bayesian Group Decision Making with Separate Aggregation of Beliefs and Values," Econometrica, 47, 1321-1336.

IPCC (2013): Climate Change 2013: The Physical Science Basis. Contribution of Working Group I to the Fifth Assessment Report of the Intergovernmental Panel on Climate Change, Cambridge, United Kingdom and New York, NY, USA: Cambridge University Press.

- (2014): Climate Change 2014: Impacts, Adaptation, and Vulnerability. Contribution of Working Group II to the Fifth Assessment Report of the Intergovernmental Panel on Climate Change, Cambridge, United Kingdom and New York, NY, USA: Cambridge University Press.

Klibanoff, P., M. Marinacci, And S. Mukerji (2005): "A Smooth Model of Decision Making under Ambiguity," Econometrica, 73, 1849-1892.

Klibanoff, P., S. Mukerui, And K. Seo (2014): "Perceived Ambiguity and Relevant Measures," Econometrica, 82, 1945-1978.

Maccheroni, F., M. Marinacci, And A. Rustichini (2006): "Ambiguity Aversion, Robustness, and the Variational Representation of Preferences," Econometrica, 74, 1447-1498.

Manski, C. (2013): Public Policy in an Uncertain World: Analysis and Decisions, Harvard University Press.

Mongin, P. (1995): "Consistent Bayesian Aggregation," Journal of Economic Theory, 66, 313351.

(1998): "The paradox of the Bayesian experts and state-dependent utility theory," Journal of Mathematical Economics, 29, 331-361. 
- (2014): "Spurious unanimity and the Pareto Principle," Economics and Philosophy, forthcoming.

Mongin, P. And M. Pivato (2015): "Ranking Multidimensional Alternatives and Uncertain Prospects," Journal of Economic Theory, 157, 146-171.

Nascimento, L. (2012): "The ex ante aggregation of opinions under uncertainty," Theoretical Economics, 7, 535-570.

Nehring, K. (2007): "Bernoulli Without Bayes: A Theory of Utility-Sophisticated Preferences under Ambiguity," Mimeo.

Pindyck, R. S. (2013): "Climate Change Policy: What Do the Models Tell Us?" Journal of Economic Literature, 51, 860-72.

Qu, X. (2015): "Separate Aggregation of Beliefs and Values under Ambiguity," Economic Theory, forthcoming.

Rigotti, L. and C. Shannon (2005): "Uncertainty and Risk in Financial Markets," Econometrica, 73, 203-243.

Rockafellar, R. T. (1970): Convex analysis, Princeton University Press.

SAvage, L. J. (1954): The Foundations of Statistics, John Wiley and Sons.

Schmeidler, D. (1989): "Subjective Probability and Expected Utility without Additivity," Econometrica, 57, 571-587.

Stern, N. (2013): "The Structure of Economic Modeling of the Potential Impacts of Climate Change: Grafting Gross Underestimation of Risk onto Already Narrow Science Models," Journal of Economic Literature, 51, 838-59.

Weymark, J. A. (1993): "Harsanyi's social aggregation theorem and the weak Pareto principle," Social Choice and Welfare, 10, 209-221.

Zuber, S. (2015): "Harsanyi's theorem without the sure-thing principle: On the consistent aggregation of Monotonic Bernoullian and Archimedean preferences," CES Working Papers, 2015-69, University of Paris 1. 